\title{
A Novel Ingestion Strategy for Sodium Bicarbonate Supplementation in a Delayed-Release Form: a Randomised Crossover Study in Trained Males
}

Nathan Philip Hilton ${ }^{* *}$ D, Nicholas Keith Leach', S. Andy Sparks ${ }^{1}$, Lewis Anthony Gough², Melissa May Craig ${ }^{3}$, Sanjoy Kumar Deb ${ }^{4}$ and Lars Robert McNaughton ${ }^{1,5}$

\begin{abstract}
Background: Sodium bicarbonate $\left(\mathrm{NaHCO}_{3}\right)$ is a well-established nutritional ergogenic aid, though gastrointestinal (Gl) distress is a common side-effect. Delayed-release $\mathrm{NaHCO}_{3}$ may alleviate $\mathrm{Gl}$ symptoms and enhance bicarbonate bioavailability following oral ingestion, although this has yet to be confirmed.

Methods: In a randomised crossover design, pharmacokinetic responses and acid-base status were compared following two forms of $\mathrm{NaHCO}_{3}$, as were Gl symptoms. Twelve trained healthy males (mean $\pm \mathrm{SD}$ age $25.8 \pm 4.5$ years, maximal oxygen uptake $\left(\dot{\mathrm{V}} \mathrm{O}_{2}\right.$ max $) 58.9 \pm 10.9 \mathrm{~mL} \mathrm{~kg} \mathrm{~min}^{-1}$, height $1.8 \pm 0.1 \mathrm{~m}$, body mass $82.3 \pm 11.1 \mathrm{~kg}$, fat-free mass $72.3 \pm 10.0 \mathrm{~kg}$ ) underwent a control (CON) condition and two experimental conditions: $300 \mathrm{mg} \mathrm{kg}^{-1}$ body mass $\mathrm{NaHCO}_{3}$ ingested as an aqueous solution (SOL) and encased in delayed-release capsules (CAP). Blood bicarbonate concentration, $\mathrm{pH}$ and base excess (BE) were measured in all conditions over $180 \mathrm{~min}$, as were subjective Gl symptom scores.

Results: Incidences of Gl symptoms and overall severity were significantly lower (mean difference $=45.1 \%, P<0.0005$ and $47.5 \%, P<0.0005$ for incidences and severity, respectively) with the CAP than with the SOL. Symptoms displayed increases at 40 to 80 min post-ingestion with the SOL that were negated with CAP $(P<0.05)$. Time to reach peak bicarbonate concentration, $\mathrm{pH}$ and $\mathrm{BE}$ were significantly longer with CAP than with the SOL.

Conclusions: In summary, CAP can mitigate Gl symptoms induced with SOL and should be ingested earlier to induce similar acid-base changes. Furthermore, CAP may be more ergogenic in those who experience severe Gl distress with SOL, although this warrants further investigation.
\end{abstract}

Keywords: Acid-base balance, Extracellular buffer, Bioavailability, Exercise-induced fatigue

\section{Key Points}

- Delayed-release $\mathrm{NaHCO}_{3}$ mitigated GI distress compared with the aqueous solution ingestion form; therefore, athletes who have experienced problematic side-effects in the past may now benefit from supplementation.

\footnotetext{
* Correspondence: hiltonn@edgehill.ac.uk

${ }^{1}$ Sports Nutrition and Performance Group, Department of Sport and Physical

Activity, Edge Hill University, St Helens Road, Ormskirk L39 4QP, UK

Full list of author information is available at the end of the article
}

- Time to reach peak blood bicarbonate increased with delayed-release $\mathrm{NaHCO}_{3}$, and therefore, requires earlier ingestion ( $48 \mathrm{~min})$ in comparison with the aqueous solution ingestion form.

- Bicarbonate bioavailability was enhanced in some individuals with delayed-release $\mathrm{NaHCO}_{3}$; hence, ingestion should be based upon individual concentration-time profiles in conjunction with GI symptoms. 


\section{Background}

Sodium bicarbonate $\left(\mathrm{NaHCO}_{3}\right)$ is a well-established nutritional ergogenic aid. Supplementation can improve short-duration ( 1-10 min), high-intensity exercise performance [1], with various meta-analyses confirming its efficacy [2-4]. As an extracellular buffering agent, $\mathrm{NaHCO}_{3}$ enhances endogenous bicarbonate buffering capacity by inducing significant, albeit transient, elevations in extracellular bicarbonate. Consequently, this enhances efflux of hydrogen cations $\left(\mathrm{H}^{+}\right)$from skeletal muscle, therefore delaying muscle fatigue and positively affecting numerous performance variables, such as power output [5] and time to exhaustion [6]. While it remains unclear whether minimal increases are required to achieve these benefits, substantial changes $(\sim 6$ $\left.\mathrm{mmol} \cdot \mathrm{L}^{-1}\right)$ in blood bicarbonate may improve the likelihood of performance-enhancing effects [2, 7]. Given that bicarbonate is lost in the neutralisation of gastric acid [8], large oral doses (200-300 $\mathrm{mg} \mathrm{kg}^{-1}$ body mass) are required to induce meaningful elevations in the blood.

Acute gastrointestinal (GI) distress is a known side-effect of ingesting large amounts of $\mathrm{NaHCO}_{3}$ [9], particularly when administered as an aqueous solution [10]. Ergogenic effects have still been observed in those reporting GI distress [1, 11]; however, there is evidence to suggest that GI distress may be ergolytic for some individuals [1, 12-14]. Furthermore, some authors have suggested that GI distress may deter individuals from using $\mathrm{NaHCO}_{3}$ regardless of its potential ergogenic benefits [7]. Although the impact of GI distress on performance remains ambiguous, symptoms such as vomiting and diarrhoea may present a major practical limitation for athletes and coaches.

Polymeric-coated compounds can resist gastric degradation and reduce GI symptoms provoked by acid sensitive compounds, such as $\mathrm{NaHCO}_{3}$ [15]. Hydroxypropyl methylcellulose, contained in delayed-release capsules, can resist degradation in acidic environments $(\mathrm{pH} \sim 1-2$ arbitrary units (AU)), and therefore, provides gastro-resistant properties. Instead, degradation occurs in the duodenum where the $\mathrm{pH}$ is far more alkaline ( $\mathrm{pH} \sim 6-7 \mathrm{AU})$ and absorption can take place rapidly. Since GI distress is partly attributable to degradation in the stomach [8], it has been suggested that gastro-resistant capsules may alleviate symptoms that are typical with $\mathrm{NaHCO}_{3}$ ingestion [16]. Given that less bicarbonate is lost in the stomach, it has also been suggested that smaller doses may produce comparable acid-base changes to larger doses [16]. In contrast, as gut transit time is reduced with gastro-resistant formulations [15], this may reduce bicarbonate bioavailability when administered in this form. No study to date has examined the use of delayed-release $\mathrm{NaHCO}_{3}$ on markers of GI distress, nor on bicarbonate bioavailability and subsequent blood acid-base responses.
Reducing GI distress following $\mathrm{NaHCO}_{3}$ ingestion may enhance use by athletes, particularly among those who are deterred by potential side-effects.

Therefore, the aim of this study was to investigate whether delayed-release $\mathrm{NaHCO}_{3}$ could mitigate GI distress compared with an aqueous solution, as well as to compare the pharmacokinetic and acid-base responses. It was hypothesised that delayed-release $\mathrm{NaHCO}_{3}$ would reduce GI symptoms and display at least bioequivalence when compared to an aqueous solution.

\section{Methods}

\section{Participants}

Twelve trained [17] healthy males (mean \pm SD age 25.8 \pm 4.5 years, maximal oxygen uptake $\left(\dot{\mathrm{VO}}_{2} \max \right) 58.9 \pm$

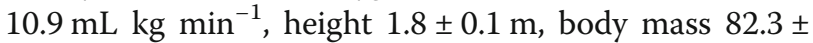
$11.1 \mathrm{~kg}$, fat-free mass $72.3 \pm 10.0 \mathrm{~kg}$ ) were recruited for the study. The study was approved by the University Research Ethics Committee (URESC) before the participants gave written informed consent to take part in the study. Inclusion in the study required that participants had performed regular ( $\geq 3$ days week $^{-1}$ ) physical exercise for at least 2 years. Exclusion criteria included ingestion of any buffering agents $<6$ months prior to commencing the study and those with hypertension or on a sodium-restricted diet.

\section{Study Overview}

Before taking part in the experimental trials, each participant underwent a baseline assessment over two laboratory visits separated by at least $48 \mathrm{~h}$ to establish (1) body composition and $\dot{\mathrm{VO}}_{2 \text { max }}$ and (2) fluctuations in blood analytes $\left(\mathrm{HCO}_{3}{ }^{-}, \mathrm{pH}\right.$ and base excess) under normal conditions. Fluctuations in blood analytes and GI symptoms under normal conditions were used as a control (CON) measure throughout. In the experimental trials, all participants underwent two conditions: $300 \mathrm{mg} \mathrm{kg}^{-1}$ body mass $\mathrm{NaHCO}_{3}$ administered as either an aqueous solution (SOL) or encased in delayed-release capsules (CAP). Experimental trials were administered in a block randomised crossover design that was counterbalanced (Latin square) in order of administration and took place at least 7 days apart to allow for the washout of residual $\mathrm{NaHCO}_{3}$ [18]. Participants were required to abstain from alcohol or caffeine-containing beverages for $12 \mathrm{~h}$ and from strenuous exercise $24 \mathrm{~h}$ before each laboratory visit. All sessions took place under standardised laboratory conditions (temperature $=21-22{ }^{\circ} \mathrm{C}$, relative humidity $=50-55 \%$, barometric pressure $=756-759 \mathrm{mmHg}$ ) and were conducted at $0900 \mathrm{~h}$ to account for circadian rhythms [19]. 


\section{Baseline Assessment}

Participants arrived at the laboratory on both occasions after an overnight fast $(\sim 12 \mathrm{~h})$ and euhydrated. On one visit, semi-nude body mass and fat-free mass were assessed using whole-body air displacement plethysmography (BOD $\mathrm{POD}^{\circ}$, COSMED, Italy). Participants then performed an incremental exercise test to volitional exhaustion on an electromagnetically braked cycle ergometer (Excalibur Sport, Lode, Netherlands). After a standardised 5-min warm-up at a power output of 70 watts $(\mathrm{W})$, the cycling protocol commenced at $75 \mathrm{~W}$ for $1 \mathrm{~min}$ and workload increased by $1 \mathrm{~W}$ every $2 \mathrm{~s}$ (30 $\mathrm{W} \mathrm{min}^{-1}$ ) until volitional exhaustion. This was determined by the inability of the participant to sustain their respective self-selected cadence for $>5 \mathrm{~s}$ despite feedback and strong verbal encouragement. On a separate visit, fingertip capillary blood samples were obtained using an aseptic technique after the participants were quietly seated for $20 \mathrm{~min}$. Blood samples were drawn every 20 min over $180 \mathrm{~min}$, with $10 \mathrm{~min}$ sampling between 80 and 140 min to accurately capture peak values [20]. Blood samples were collected in 100- $\mu \mathrm{L}$ heparin-coated glass clinitubes (Radiometer Medical Ltd., Denmark) and immediately analysed using a blood gas analyser (ABL800 BASIC, Radiometer Medical Ltd., Denmark). At the same time points, GI symptoms were recorded using a 9-item questionnaire, including nausea, flatulence, stomach cramping, belching, stomach ache, bowel urgency, diarrhoea, vomiting and stomach bloating [10]. Symptoms were self-measured on a $10-\mathrm{cm}$ scale, the ends of which were marked " 0 , no symptom" and " 10 , severe symptom", as previously described [11]. Participants remained seated throughout, although toilet breaks were permitted. No food was consumed during the experimental trials, and water was permitted ad libitum, with volumes replicated in the subsequent experimental session.

\section{Experimental Trials}

Treatment condition SOL was prepared in $400 \mathrm{~mL}$ of natural mineral water (Evian', France) and mixed with $50 \mathrm{~mL}$ of sugar-free blackcurrant flavoured squash (Robinsons $\left.{ }^{\circ}, \mathrm{UK}\right)$ and refrigerated $(\sim 1 \mathrm{~h})$ to enhance palatability [11]. For the CAP condition, size 00 capsules $\left(\right.$ DRcaps $^{\mathrm{mu}}$, Capsugel ${ }^{\circ}$, France) were prefilled with $\mathrm{NaHCO}_{3}$ using a capsule filler (Capsule Connection LLC, USA), while doses were checked for accuracy using digital laboratory scales (Fisher, OHAUS ${ }^{\mathrm{Tm}}$ ). Participants were instructed to ingest either the SOL or CAP with an equal volume $(400 \mathrm{~mL})$ of water within $10 \mathrm{~min}$ while the stopwatch commenced parallel with the start of ingestion $[20,21]$. All experimental trials were conducted under the same conditions as the CON trial, and blood analytes and GI symptoms were measured as previously described.

\section{Statistical Analysis}

Prospective statistical power analysis was conducted a priori to determine that 12 participants were required, with alpha and beta set at 0.05 and 0.20 , respectively. Data were assessed for normality using standard graphical methods prior to analyses [22]. Two-way analysis of variance (ANOVA) with repeated measures (condition $x$ time) was used to establish significant main effects for blood analytes $\left(\mathrm{HCO}_{3}{ }^{-}, \mathrm{pH}\right.$ and $\left.\mathrm{BE}\right)$ and GI symptom scores. Condition consisted of two levels (SOL and CAP), whereas time consisted of $13(0,20,40,60,80$, 90, 100, 110, 120, 130,140, 160 and $180 \mathrm{~min})$. Effect sizes were calculated using partial eta squared $\left(\eta^{2}\right)$ for ANOVA and were interpreted according to Cohen [23] as follows: trivial $<0.20$, small $0.20-0.49$, moderate $0.50-0.79$ and large $\geq 0.80$. Blood analytes and GI symptom scores were then analysed using one-way ANOVA to establish differences at individual time points. Sphericity was assessed using Mauchly's test throughout. Where appropriate, corrections for violations of sphericity (Greenhouse-Geisser) and multiple comparisons of differences within a factor (Bonferroni) were made [24]. Mean pharmacokinetic variables and highest GI symptom score between conditions were analysed by paired-sample $t$ test. Descriptive data are presented as mean \pm SD unless stated otherwise. The $\alpha$-level of statistical significance was set at $P<0.05$, and exact $P$ values are given in the text and tables. Values for $P$ of " 0.000 " given by the statistical package were corrected to "<0.0005" [25]. Data were analysed using the Statistical Package for the Social Sciences $\left(\right.$ SPSS $^{\circ}$ ) for Windows ${ }^{\circ}$ (IBM, Chicago, IL, USA), version 25.

\section{Results}

\section{Gastrointestinal Distress}

No GI symptoms were reported pre-ingestion, nor at any time point in the $\mathrm{CON}$ condition. All participants $(N=12)$ experienced at least one GI symptom following SOL and CAP ingestion (Table 1). Stomach bloating was the most prevalent GI symptom in both experimental trials, although this was lower with CAP (58\%) than with SOL (100\%). Overall, fewer GI symptoms (mean difference $=45.1 \%$ ) were reported with CAP than with SOL (Fig. 1a). Incidences of GI distress peaked at $40 \mathrm{~min}$ post-ingestion under both conditions (Fig. 1a), which was predominantly due to belching and bowel urgency.

Overall GI symptoms increased in the SOL $(P<0.0005)$ and CAP $(P<0.017)$ conditions beyond those observed in the CON condition. There was a significant effect of ingestion form $\left(F_{1.00,11.00}=21.13, P=0.001, \eta^{2}=0.66\right)$, with less severe GI symptoms reported with CAP than with the 
Table 1 The most severe individual Gl symptom reported during any trial. Symptom scores are displayed in parenthesis and are expressed as arbitrary units (AU)

\begin{tabular}{llll}
\hline Participant & CON & SOL & CAP \\
\hline 1 & Nil (0.0) & Stomach cramp (3.5) & Stomach bloating (3.0) \\
2 & Nil (0.0) & Bowel urgency (7.0) & Stomach bloating (3.0) \\
3 & Nil (0.0) & Nausea (6.0) & Nausea (2.0) \\
4 & Nil (0.0) & Diarrhoea (10.0) & Stomach bloating (7.0) \\
5 & Nil (0.0) & Diarrhoea (10.0) & Diarrhoea (7.0) \\
6 & Nil (0.0) & Diarrhoea (10.0) & Diarrhoea (5.5) \\
7 & Nil (0.0) & Bowel urgency (6.0) & Bowel urgency (5.0) \\
8 & Nil (0.0) & Bowel urgency (10.0) & Bowel urgency (2.0) \\
9 & Nil (0.0) & Diarrhoea (10.0) & Belching (3.0) \\
10 & Nil (0.0) & Diarrhoea (10.0) & Belching (3.0) \\
11 & Nil (0.0) & Stomach ache (3.0) & Belching (3.0) \\
12 & Nil (0.0) & Diarrhoea (10.0) & Diarrhoea (7.0) \\
Mean (SD) & $0.00 \pm 0.00$ & 7.96 \pm 2.73 AU & 4.21 \pm 1.97 AU \\
\hline
\end{tabular}

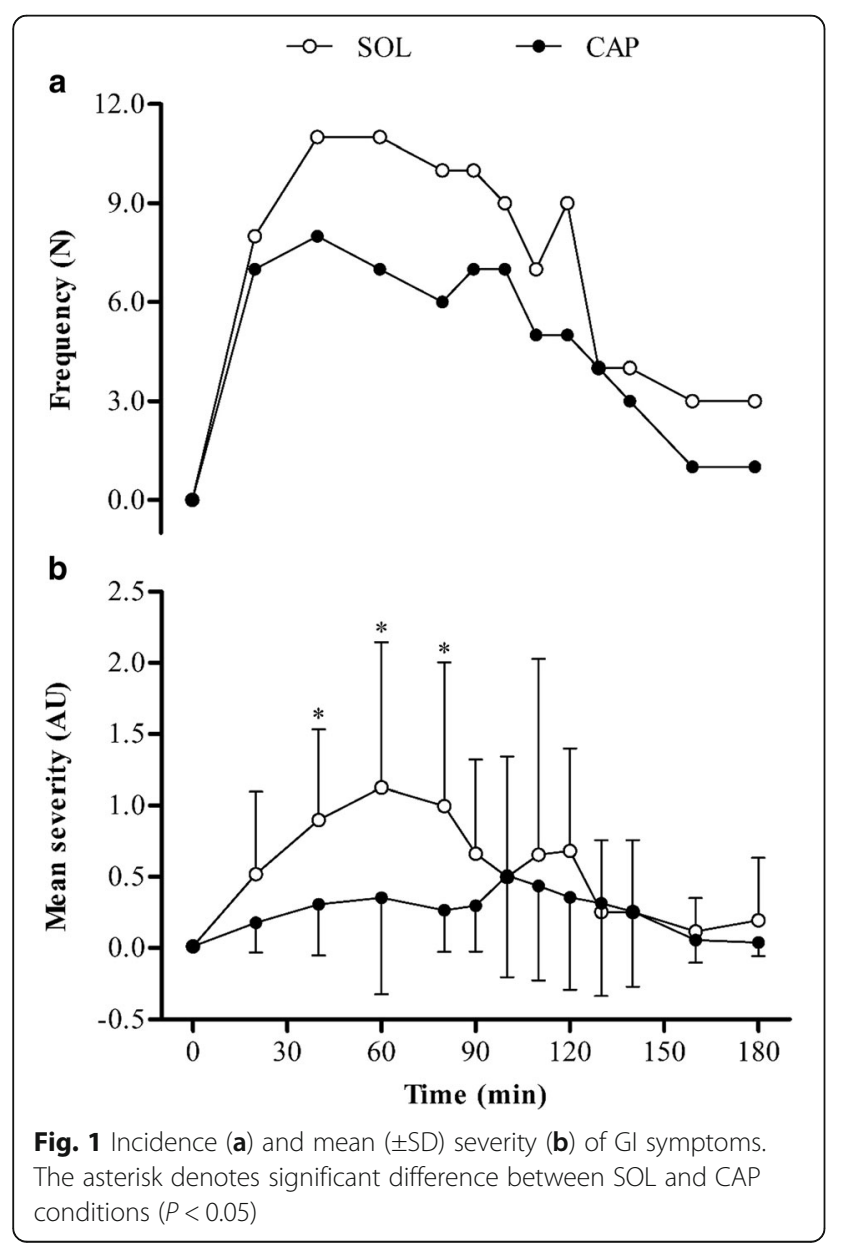

SOL $(P=0.001)$ (Fig. $1 \mathrm{~b})$. There was no effect of time $\left(F_{2.85}\right.$, $\left.31.36=2.89, P=0.053, \eta^{2}=0.21\right)$, although symptoms at 40 min were significantly greater than pre-ingestion $(P=0.03)$. No significant interaction was found $\left(F_{3.22}, 35.39=1.87, P=\right.$ $\left.0.148, \eta^{2}=0.15\right)$. Overall GI symptoms were significantly greater with the SOL at $20(P=0.004), 40(P<0.0005), 60$ $(P=0.002), 80 \quad(P=0.001), 90 \quad(P=0.002)$ and $120(P=$ $0.018)$ min post-ingestion than in the $\mathrm{CON}$ condition. Symptoms were significantly lower at $40(P=0.004), 60(P$ $=0.035)$ and $80(P=0.017)$ min post-ingestion with CAP than with the SOL. Gastric symptoms were significantly lower at $40(P=0.006), 60(P=0.020)$ and $80(P=0.021)$ min post-ingestion with CAP than with the SOL, while no significant differences were reported for intestinal symptoms $(P>0.05)$. There was a significant difference in the most severe GI symptom experienced in the SOL (7.21 \pm $2.48 \mathrm{AU})$ and CAP $(4.29 \pm 2.12 \mathrm{AU})$ conditions $(P=0.002)$, respectively (Table 1 ). Time to reach the most severe individual GI symptom was greater with the SOL $(87.50 \pm$ $50.29 \mathrm{~min})$ than with CAP $(75.00 \pm 32.33 \mathrm{~min})$, although these were not significant $(P>0.05)$.

\section{Bicarbonate Bioavailability}

Ingestion form had no significant effect on bicarbonate concentration $\left(F_{1.00,11.00}=0.71, P>0.05, \eta^{2}=0.061\right)$ up to $180 \mathrm{~min}$ post-ingestion. There was a significant effect of time $\left(F_{2.38}, 26.23=101.74, P<0.0005, \eta^{2}=0.90\right)$; bicarbonate concentration increased notably for $60 \mathrm{~min}$ following ingestion of the SOL, until a decrease occurred from the previous time point at $180 \mathrm{~min}(P=0.004)$ post-ingestion (Fig. 2). In the CAP condition, bicarbonate concentration rose progressively between 40 and 90 min, after which bicarbonate did not significantly change $(P>0.05)$. A significant interaction was found between

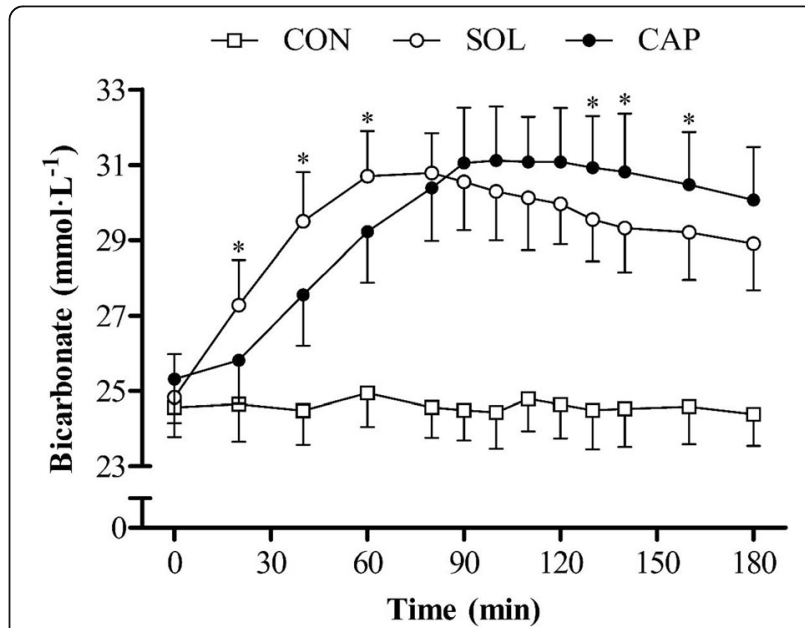

Fig. 2 Mean $( \pm S D)$ blood bicarbonate concentrations. The asterisk denotes significant difference between $\mathrm{SOL}$ and CAP conditions $(P<0.05)$ 
condition and time $\left(F_{2.31}, 25.44=16.48, P<0.0005, \eta^{2}\right.$ $=0.60)$. Bicarbonate concentrations were significantly higher with the SOL at $20(P=0.008), 40(P=0.001)$ and $60(P=0.011) \mathrm{min}$ post-ingestion than with the CAP and significantly lower at $130(P=0.021), 140$ $(P=0.019)$ and $160 \quad(P=0.047) \mathrm{min}$ post-ingestion. Mean pharmacokinetic variables were similar between conditions (Table 2). There was a delay in the absorption of bicarbonate with CAP; lag time $\left(T_{\mathrm{lag}}\right)$ was greater with CAP than with SOL $(P=0.002)$, as was the time to reach peak bicarbonate concentration $(P<0.0005)$. Peak bicarbonate concentration $\left(C_{\max }\right)$, change in bicarbonate concentration $\left(\Delta C_{\max }\right)$ and area under the curve $\left(\mathrm{AUC}_{0-3 \mathrm{~h}}\right)$ increased in the SOL and CAP conditions $(P<0.005)$ compared with the $\mathrm{CON}$, with no significant differences between conditions $(P>0.05)$. However, a greater number of participants reached a $5 \mathrm{mmol} \mathrm{L}^{-1}$ (SOL $N=10$, CAP $N=11)$ and $6 \mathrm{mmol} \mathrm{L}^{-1}(\mathrm{SOL} N=8$, CAP $N=9$ ) increase in bicarbonate with CAP than with the SOL (Fig. 3).

\section{Acid-Base Balance}

Ingestion form had no significant effect on $\mathrm{pH}\left(F_{1.00}\right.$, $\left.11.00=2.88, \quad P>0.05, \quad \eta^{2}=0.21\right)$ up to $180 \mathrm{~min}$ post-ingestion. There was a significant effect of time $\left(F_{4.42,} 48.60=43.74, P<0.0005, \eta^{2}=0.80\right) ; \mathrm{pH}$ increased markedly for $60 \mathrm{~min}$ following ingestion of the SOL, until a decrease occurred from the previous time point at $180 \mathrm{~min}(P=0.004)$ post-ingestion (Fig. 4$)$. In the CAP condition, $\mathrm{pH}$ rose progressively between 40 and $90 \mathrm{~min}$, after which $\mathrm{pH}$ did not significantly change $(P>0.05)$. A significant interaction was found between condition and time for $\mathrm{pH}\left(F_{4.88}, 53.67=6.42, P<0.0005, \eta^{2}=0.37\right)$. Blood $\mathrm{pH}$ was significantly higher with the SOL at 40 $\min (P=0.009)$ post-ingestion than with the CAP and significantly lower at $120 \mathrm{~min}(P=0.017)$ post-ingestion. Blood $\mathrm{pH}$ peaked much later with the $\mathrm{CAP}(\mathrm{SOL}=$ $71.67 \pm 25.88 \mathrm{~min}, \mathrm{CAP}=125.83 \pm 27.75 \mathrm{~min}, P=0.001$ ) than with the SOL, although absolute changes were comparable between conditions $(P=0.093)$.

Similarly, ingestion form had no significant effect on base excess $(\mathrm{BE})\left(F_{1.00}, 11.00=0.69, P>0.05, \eta^{2}=0.06\right)$ up to $180 \mathrm{~min}$ post-ingestion. There was a significant effect

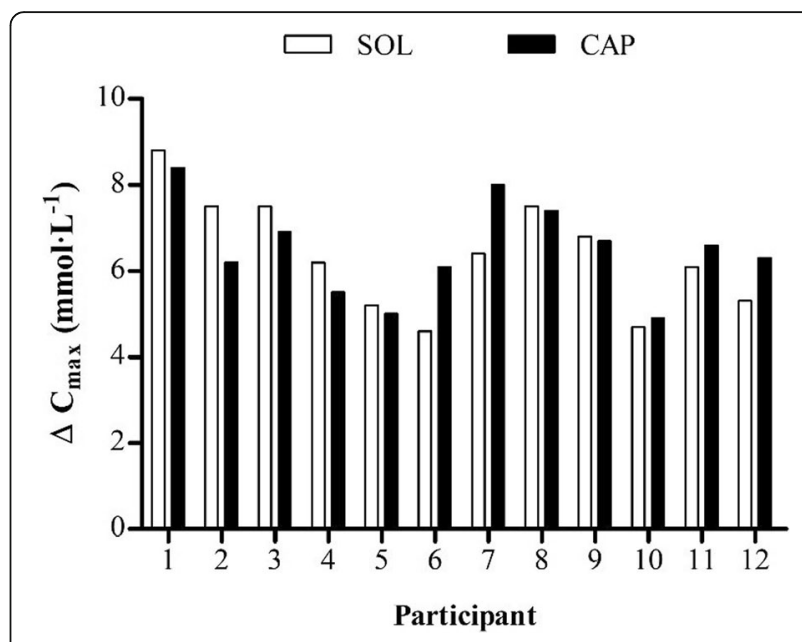

Fig. 3 Individual changes in blood bicarbonate concentration between conditions (SOL and CAP)

of time $\left(F_{2.24,24.68}=118.08, P<0.0005, \eta^{2}=0.92\right)$; $\mathrm{BE}$ increased markedly for $60 \mathrm{~min}$ following ingestion of the SOL, until a decrease occurred from the previous time point at $180 \mathrm{~min}(P=0.034)$ post-ingestion (Fig. 4). In contrast, BE rose progressively between 40 and $90 \mathrm{~min}$ in the CAP condition, after which BE did not significantly change $(P>0.05)$. A significant interaction was found between condition and time $\left(F_{2.20,24.18}=15.35\right.$, $\left.P<0.0005, \quad \eta^{2}=0.58\right)$. Blood $\mathrm{BE}$ was significantly higher with the SOL at $20(P=0.014), 40(P=0.005)$ and $60(P=0.034) \mathrm{min}$ post-ingestion than with the CAP and significantly lower at $130(P=0.022)$ and $140(P=0.019) \mathrm{min}$ post-ingestion. Blood BE peaked much later with $\mathrm{CAP}(\mathrm{SOL}=71.67 \pm 18.01 \mathrm{~min}$, CAP $=112.50 \pm 27.01 \mathrm{~min}, P<0.0005)$ than with the SOL, although absolute changes were comparable between conditions $(P=0.071)$.

\section{Discussion}

This is the first study to investigate the effects of gastro-resistant capsules on GI distress, bicarbonate bioavailability and subsequent acid-base responses following $\mathrm{NaHCO}_{3}$ ingestion. The main finding was that

Table 2 Mean $( \pm S D$ ) pharmacokinetic response variables for bicarbonate in the SOL and CAP conditions, together with the statistical significance of the difference

\begin{tabular}{lllll}
\hline Outcome & SOL & CAP & $t$ test & $P$ value \\
\hline$T_{\text {lag }}(\min )$ & $20.0 \pm 0.0^{*}$ & $31.7 \pm 10.3^{*}$ & -3.92 & 0.002 \\
$T_{\max }(\min )$ & $71.7 \pm 18.0^{* *}$ & $120.0 \pm 28.9^{* *}$ & -5.35 & $<0.0005$ \\
$C_{\max }(\mathrm{mmol} \mathrm{L}$ & -1.66 & 0.125 \\
$\Delta C_{\max }\left(\mathrm{mmol} \mathrm{L}^{-1}\right)$ & $31.2 \pm 1.1$ & $31.8 \pm 1.3$ & -0.46 & 0.658 \\
$\mathrm{AUC}$ & $6.4 \pm 1.3$ & $6.5 \pm 1.1$ & -0.13 & 0.899 \\
\hline
\end{tabular}

The asterisk denotes significant difference between SOL and CAP $\left({ }^{*} P<0.05,{ }^{* *} P<0.0005\right) . T_{\text {lag }}$ time to commence change in bicarbonate concentration, $T_{\text {max }}$ time to peak concentration, $C_{\max }$ peak bicarbonate concentration, $\Delta C_{\max }$ absolute change in bicarbonate concentration, $A U C_{0-3 h}$ area under the concentration-time curve 


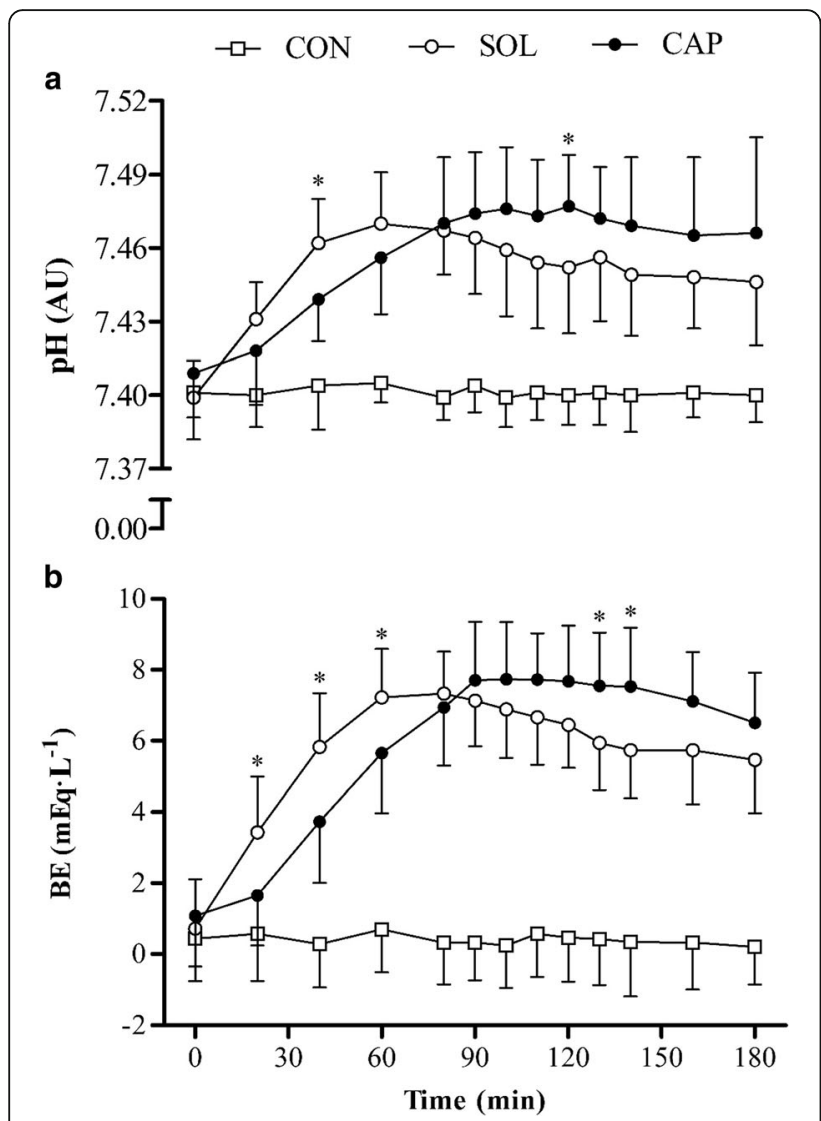

Fig. 4 Mean $( \pm S D) p H(\mathbf{a})$ and $B E(\mathbf{b})$ responses. The asterisk denotes significant difference between SOL and CAP conditions $(P<0.05)$

delayed-release $\mathrm{NaHCO}_{3}$ mitigated GI distress, as hypothesised. Fewer GI symptoms $(\sim 45.1 \%)$ were reported with the delayed-release capsules, and the overall severity was reduced $(\sim 47.1 \%)$ when compared to the aqueous solution. Interestingly, reductions in GI symptoms were due to gastric but not intestinal symptoms, a finding that has been suggested in the relevant literature [15]. Gastrointestinal symptoms were negated with the delayed-release capsules, with a reduction in the most severe symptom experienced up to $3 \mathrm{~h}$ following supplementation (Table 1). Given that GI symptoms may be ergolytic $[13,14,26]$, delayed-release $\mathrm{NaHCO}_{3}$ may be more ergogenic in those who experience severe GI distress with the aqueous solution. Furthermore, since GI distress may deter some individuals from using $\mathrm{NaHCO}_{3}$ as an ergogenic aid [7, 10], delayed-release $\mathrm{NaHCO}_{3}$ would appear to be a more favourable option for athletes and coaches.

While necessary to achieve erogenicity [27], large boluses ( 200-300 mg kg-1 body mass) of $\mathrm{NaHCO}_{3}$ can induce significant GI symptoms. In the current study, there was a high incidence of GI distress with the aqueous solution, which is in agreement with some authors [13] but not others [10, 28]. Symptoms are considered to have both gastric and intestinal causes [8], a finding that is supported by the current study. On entering the stomach, $\mathrm{NaHCO}_{3}$ dissociates to sodium and bicarbonate ions, the latter of which produces carbon dioxide during the neutralisation of gastric acid [8]. Consequently, carbon dioxide tension increases exponentially with exposure and is associated with gastric symptoms, such as belching, nausea and stomach ache. Intestinal symptoms, though partly induced from elevated carbon dioxide tension in the intestinal lumen, originate from excess sodium that aggravates the intestinal mucosa and creates osmotic fluctuations leading to bowel urgency and diarrhoea [13]. Delayed-release capsules, partly formulated with a polymeric barrier, have gastro-resistant properties and can minimise disintegration in the stomach. Mitigating gastric symptoms may indeed have implications for performance. Previous research indicates that symptoms can inhibit high-intensity cycling performance [14], while others have reported improvements irrespectively [26]. Since numerous participants have withdrawn from studies due to GI distress [29], previous research may have underestimated the ergolytic effect of such symptoms. Studies that have attempted to mitigate GI symptoms following $\mathrm{NaHCO}_{3}$ ingestion have done so using alternative dosing strategies. Gelatine capsules co-ingested with a small high-carbohydrate $\left(1.5 \mathrm{~g} \mathrm{~kg}^{-1}\right.$ body mass) meal are currently regarded as the formulation least likely to induce GI symptoms following $\mathrm{NaHCO}_{3}$ ingestion [10]. In the current study, delayedrelease capsules were ingested after an overnight fast, largely to minimise potential confounding effects of food on acid-base changes. Nevertheless, co-ingestion with a small high-carbohydrate meal may have further reduced GI symptoms and warrants further investigation. Furthermore, while comparison with an aqueous solution was chosen based on its frequency of use within the literature, this may not be the case in the practice and is thus a limitation to the study. Future work should look to assess the pharmacokinetics of $\mathrm{NaHCO}_{3}$ administered based on capsule composition only, so that the mechanism underpinning bioavailability and reductions in GI distress may be better understood.

In relation to bioavailability, both ingestion forms provided adequate sources of bicarbonate and displayed similar pharmacokinetic properties. Increases in bicarbonate were comparable, with both forms exceeding the $6 \mathrm{mmol} \mathrm{L}^{-1}$ threshold suggested to enhance ergogenicity [2]. Interestingly, some $(N=3)$ participants displayed enhanced bicarbonate availability $\left(\geq 1 \mathrm{mmol} \mathrm{L^{-1 }}\right)$ with delayed-release capsules (Fig. 4), while only one participant was found to have enhanced bicarbonate availability of this magnitude with the aqueous solution. Similarly, more participants achieved a $5 \mathrm{mmol} \mathrm{L}^{-1}(\mathrm{SOL} N=10$, CAP $N=11)$ and $6 \mathrm{mmol} \mathrm{L}^{-1}(\mathrm{SOL}: N=8$; CAP: $N=9)$ 
increase in bicarbonate with the delayed-release capsules than with the aqueous solution. These results would be explained by the gastric bypass model proposed by Oliveira et al. [16], which includes the effect of gastric transit time, and bicarbonate loss associated with neutralisation. As suggested by these authors, reducing bicarbonate neutralisation in the stomach increases bioavailability when $\mathrm{NaHCO}_{3}$ is administered orally. Since changes of $\sim 1 \mathrm{mmol} \mathrm{L}^{-1}$ in bicarbonate concentration can positively affect performance [26], delayed-release $\mathrm{NaHCO}_{3}$ may elicit superior ergogenicity. In contrast to the aqueous solution, bicarbonate absorption did not commence immediately following capsule ingestion, suggesting that the delayed-release capsules were effective $[15,30]$. This result indicates that the capsules achieved disintegration in the intestine, which had the effect of lengthening (+48.3 $\mathrm{min})$ the time to reach peak bicarbonate concentration. Bicarbonate peaked at $\sim 120 \mathrm{~min}$ post-ingestion with the delayed-release capsules, which is later than previously reported with an aqueous solution in some studies [20] but not all [21]. Similar to previous studies [31-33], there was a high degree of individual variability in the time to reach peak bicarbonate concentration, although this was greater with the capsules. The current findings for bicarbonate indicate that for most individuals, delayed-release $\mathrm{NaHCO}_{3}$ may increase the likelihood of inducing a performance-enhancing effect; however, as this was not consistent for all individuals, decisions around ingestion form should be based upon individual concentration-time profiles.

Metabolic alkalosis was induced earlier with the aqueous solution $(\sim 40 \mathrm{~min})$ than with delayed-release capsules $(\sim 60 \mathrm{~min})$, although this state was maintained for longer $(+30 \mathrm{~min})$ when ingested in the delayed-release form. Homeostatic regulation, through respiratory compensation [34], may have been stimulated to a lesser extent with slower bicarbonate absorption, rather than the abrupt elevation observed with an aqueous solution. Exercise performance timed with peak alkalosis may enhance the ergogenicity of $\mathrm{NaHCO}_{3}$ [7, 29]; therefore, it is reasonable to consider that delayed-release may provide a larger ergogenic window. In a competitive setting, this may be more favourable since performance may not commence parallel with peak alkalosis due to variable factors, such as sports fixtures. In the current study, time to reach peak alkalosis was much later with delayed-release $\mathrm{NaHCO}_{3}(\sim 125 \mathrm{~min})$ than with the aqueous solution $(\sim 72 \mathrm{~min})$, with one participant peaking at $180 \mathrm{~min}$ post-ingestion. In a practical setting, delayed-release $\mathrm{NaHCO}_{3}$ would have to be ingested sooner than an aqueous solution to elicit similar acid-base changes. This may be favourable in terms of ergogenicity since GI symptoms were negligible at later time points. In contrast, given that bicarbonate concentrations were significantly lower with delayed-release $\mathrm{NaHCO}_{3}$ up to 60 min post-ingestion, this form may be less ergogenic when ingested less than $60 \mathrm{~min}$ prior to exercise.

\section{Conclusions}

In summary, delayed-release $\mathrm{NaHCO}_{3}$ mitigates GI symptoms and these effects do not reflect the intestinal component but rather the gastric component of overall symptoms. The similar pharmacokinetic properties, coupled with a delay in the time to reach metabolic alkalosis, mean that delayed-release $\mathrm{NaHCO}_{3}$ requires earlier ingestion than with an aqueous solution to induce comparable acid-base changes. The current study supports the gastric bypass model, which can be used as a model for exploring various ingestion forms and modes of administration generally. Lastly, delayed-release $\mathrm{NaHCO}_{3}$ may be more ergogenic in those who experience severe gastrointestinal distress with an aqueous solution, although this warrants further investigation.

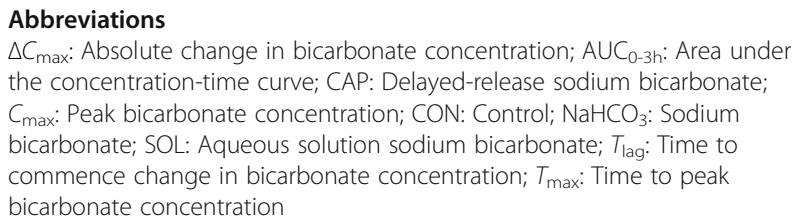

\section{Acknowledgements}

We thank all those who gave their time to participate in the study, and for the technical support of Scott Mosher and Ashleigh Warner. We thank

Capsugel $^{\oplus}$ (France) who kindly provided us with the capsules (DRcaps ${ }^{\top M}$ ) free of charge.

\section{Funding}

No funding was received for this study.

\section{Availability of Data and Materials}

Supporting data is available upon request (see corresponding author email).

\section{Authors' Contributions}

The study was designed by NPH, LRM and AS. Data were collected by NPH, $N K L$ and LAG. The manuscript was written by NPH, with feedback provided by LRM, AS and MMC. All authors approved the final version of the manuscript.

\section{Ethics Approval and Consent to Participate}

Ethical approval was granted by the University Research Ethics Committee (URESC17-NH01), and each participant provided informed consent prior to commencing the study (see methods section).

\section{Consent for Publication}

Each participant provided informed consent for the results of this study to be published.

\section{Competing Interests}

The authors, Nathan Philip Hilton, Nicholas Keith Leach, S. Andy Sparks, Lewis Anthony Gough, Melissa May Craig, Sanjoy Kumar Deb, and Lars Robert McNaughton, declare that they have no competing interests.

\section{Publisher's Note}

Springer Nature remains neutral with regard to jurisdictional claims in published maps and institutional affiliations. 


\section{Author details}

${ }^{1}$ Sports Nutrition and Performance Group, Department of Sport and Physical Activity, Edge Hill University, St Helens Road, Ormskirk L39 4QP, UK. ${ }^{2}$ School of Health Sciences, Birmingham City University, Birmingham, UK. ${ }^{3}$ Therapies Department, Liverpool Heart and Chest Hospital NHS Foundation Trust, Liverpool, UK. ${ }^{4}$ School of Life Sciences, University of Westminster, London, UK. ${ }^{5}$ Department of Sport and Movement Studies, Faculty of Health Science, University of Johannesburg, Johannesburg, South Africa.

Received: 24 October 2018 Accepted: 14 January 2019

Published online: 24 January 2019

\section{References}

1. McNaughton LR, Gough L, Deb S, Bentley D, Sparks SA. Recent developments in the use of sodium bicarbonate as an ergogenic aid. Curr Sports Med Rep. 2016;15:233-44.

2. Carr AJ, Hopkins WG, Gore CJ. Effects of acute alkalosis and acidosis on performance: a meta-analysis. Sports Med. 2011;41:801-14.

3. Christensen PM, Shirai Y, Ritz C, Nordsborg NB. Caffeine and bicarbonate for speed. A meta-analysis of legal supplements potential for improving intense endurance exercise performance. Front Physiol. 2017;8:240-56.

4. Matson LG, Tran ZV. Effects of sodium bicarbonate ingestion on anaerobic performance: a meta-analytic review. Int J Sport Nutr. 1993;3:2-28.

5. Bellinger PM, Howe ST, Shing CM, Fell JW. Effect of combined $\beta$-alanine and sodium bicarbonate supplementation on cycling performance. Med Sci Sports Exerc. 2012;44:1545-51.

6. Higgins MF, James RS, Price MJ. The effects of sodium bicarbonate (NaHCO3) ingestion on high intensity cycling capacity. J Sports Sci. 2013;31: 972-81.

7. Heibel AB, Perim PHL, Oliveira LF, McNaughton LR, Saunders B. Time to optimize supplementation: modifying factors influencing the individual responses to extracellular buffering agents. Front Nutr. 2018;5:1-12.

8. Turnberg LA, Fordtran JS, Carter NW, Rector FC. Mechanism of bicarbonate absorption and its relationship to sodium transport in the human jejunum. J Clin Invest. 1970;49:548-56.

9. Burke LM, Pyne DB. Bicarbonate loading to enhance training and competitive performance. Int J Sports Physiol Perform. 2007:2:93-7.

10. Carr AJ, Slater GJ, Gore CJ, Dawson B, Burke LM. Effect of sodium bicarbonate on [HCO3-], pH, and gastrointestinal symptoms. Int J Sport Nutr Exerc Metab. 2011;21:189-94

11. Miller P, Robinson AL, Sparks SA, Bridge CA, Bentley DJ, McNaughton LR. The effects of novel ingestion of sodium bicarbonate on repeated sprint ability. J Strength Cond Res. 2016;30:561-8.

12. Cameron SL, Mclay-Cooke RT, Brown RC, Gray AR, Fairbairn KA. Increased blood $\mathrm{pH}$ but not performance with sodium bicarbonate supplementation in elite rugby union players. Int J Sport Nutr. 2010;20:307-21.

13. Kahle LE, Kelly PV, Eloit KA, Weiss EP. Acute sodium bicarbonate loading has negligible effects on resting and exercise blood pressure but causes gastrointestinal distress. Nutr Res. 2013;33:479-86.

14. Saunders B, Sale C, Harris RC, Sunderland C. Sodium bicarbonate and highintensity-cycling capacity: variability in responses. Int J Sports Physiol Perform. 2014;9:627-32.

15. Barbosa JAC, Conway BR, Merchant HA. Going natural: using polymers from nature for gastroresistant applications. Br J Pharm. 2017;2:14-30.

16. Oliveira LF, Saunders B, Artioli GG. Is bypassing the stomach a means to optimize sodium bicarbonate supplementation? A case study with a postbariatric surgery individual. Int J Sport Nutr Exerc Metab. 2018;26:1-4.

17. DePauw K, Roelands B, Cheung SS, de Geus B, Rietjens G, Meeusen R. Guidelines to classify subject groups in sport-science research. Int I Sports Physiol Perform. 2013;8:111-22.

18. Bishop D, Edge J, Davis C, Goodman C. Induced metabolic alkalosis affects muscle metabolism and repeated-sprint ability. Med Sci Sports Exerc. 2004; 36:807-13.

19. Reilly T. Human circadian rhythms and exercise. Crit Rev Biomed Eng. 1990; 18:165-80.

20. Gough LA, Deb SK, Sparks SA, McNaughton LR. The reproducibility of blood acid base responses in male collegiate athletes following individualised doses of sodium bicarbonate: a randomised controlled crossover study. Sports Medi. 2017;47:2117-27.

21. Stannard RL, Stellingwerff T, Artioli GG, Saunders B, Cooper S, Sale C. Doseresponse of sodium bicarbonate ingestion highlights individuality in time course of blood analyte responses. Int J Sports Nutr Exerc Metab. 2016;26: 445-53.

22. Grafen A, Hails R. Modern statistics for the life sciences. Oxford: Oxford University Press; 2002.

23. Cohen J. Statistical power analysis for the behavioural sciences. 2nd ed. Hillsdale: Erlbaum; 1988.

24. Atkinson G. Analysis of repeated measurements in physical therapy research: multiple comparisons amongst level means and multi-factorial designs. Phys Ther Sport. 2002;3:191-03.

25. Kinnear PR, Gray LTD. SPSS for windows made simple. London: Lawrence Erlbaum; 1995.

26. McNaughton LR. Bicarbonate ingestion: effects of dosage on 60 s cycle ergometry. J Sports Sci. 1992;10:415-23.

27. Jones RL, Stellingwerff T, Artioli GG, Saunders B, Cooper S, Sale C. Doseresponse of sodium bicarbonate ingestion highlights individuality in time course of blood analyte responses. Int J Sport Nutr Exerc Metab. 2016;26: 445-53.

28. Montfoort MC, Van Dieren L, Hopkins WG, Shearman JP. Effects of ingestion of bicarbonate, citrate, lactate, and chloride on sprint running. Med Sci Sports Exerc. 2004;36:1239-43.

29. Gough LA, Deb SK, Sparks SA, McNaughton LR. The reproducibility of 4-km time trial (TT) performance following individualised sodium bicarbonate supplementation: a randomised controlled trial in trained cyclists. Sports Med. 2017;3:34-44.

30. Marzorati M, Possemiers S, Verhelst A, Cadé A, Madit N, Van de Wiele T. A novel hypromellose capsule, with acid resistance properties, permits the targeted delivery of acid-sensitive products to the intestine. LWT Food Sci Technol. 2015;60:544-51.

31. Renfree $\mathrm{A}$. The time course of changes in plasma $[\mathrm{H}+]$ after sodium bicarbonate ingestion. Int J Sports Physiol Perform. 2007;2:323-6.

32. Siegler JC, Marshall PW, Bray J, Towlson C. Sodium bicarbonate supplementation and ingestion timing: does it matter? J Strength Cond Res. 2012:26:1953-8.

33. Siegler JC, Midgley AW, Polman RC, Lever R. Effects of various sodium bicarbonate loading protocols on the time-dependent extracellular buffering profile. J Strength Cond Res. 2010;24:2551-7.

34. Atherton JC. Acid-base balance: maintenance of plasma pH. Anaesth Intensive Care Med. 2009;26:557-61.

\section{Submit your manuscript to a SpringerOpen ${ }^{\circ}$ journal and benefit from:}

- Convenient online submission

- Rigorous peer review

- Open access: articles freely available online

- High visibility within the field

- Retaining the copyright to your article

Submit your next manuscript at $>$ springeropen.com 